\title{
Resolution of Some problems in the Identity Theory of Mind
}

\author{
Shanjendu Nath \\ Associate Professor, Department of Philosophy, Rabindrasadan Girls' College, Karimganj, Assam, India.
}

\begin{abstract}
The identity theory of mind came into existence as a reaction to the theory of Behaviourism. This theory is advocated and developed by different philosophers beginning with Place, Feigl and Smart. According to this theory, certain physical states of brain are identical to mental states. In others words, this theory holds that the so-called mental phenomena like thoughts, feelings, wishes and the rest are identical with the bodily states and processes. Thus to have some specific kind of thought is to have some kind of specific states and processes of bodily cells, typically brain cells. When we say that someone is in a certain mental state, it implies that in the cerebral cortex of the brain of that person, certain physical event is going on. The person concerned may not be aware of the happenings of the brain but these two states are not merely correlated with each other rather these two are one and the same event in the literal sense. Thus this theory asserts that everything mental is physical. But though it speaks of mental states it does not assert that these are not physical.

Although this theory is better than dualism and Behaviourism, still it has its own problems. These problems arethe problem of identity, the problem of co-existence and the problem of consciousness. But in this paper I will discuss the problem of identity and the problem of co-existence and subsequently efforts will be made to solve these problems from materialist point of view.
\end{abstract}

Keywords: Brain, Co-existence, Identity, materialist, Mind.

\section{Introduction}

The problem of mind-body relation is very old in the history of philosophy. Different philosophers have given different theories for the solution of this problem but none is universally accepted. Among the theories, the identity theory of mind occupies an important place in explaining this problem.

The Identity Theory of mind came into existence as a serious philosophical thesis in the late 1950's. Although this theory was introduced by the psychologist E. G. Boring in 1933, it took a long duration of time to be accepted as an alternative theory in philosophy. The pioneering works which deserve credit for the acceptance of the philosophical version of this theory in philosophical domain were - U. T. Place's "Is Consciousness A Brain Process?" (1956), Herbert Feigl's "The "Mental" and the "Physical" (1958) and in 1959 J. J. C. Smart's paper "Sensations and Brain Processes" (1959). Of these three papers, the earliest one was U. T. Place's. Thus, he may rightly be said to be the fore runner of this theory next to Boring. All of these papers were depending on the same basic position although the differences of them were remarkable in their details. But in spite of their differences the area of agreement was sufficiently greater than that of their differences.

Being materialistic, this theory explained the problem and has given solution in a simple way and more scientifically. The basic theme of the theory is -mental states and processes are identical with brain states and processes. But in spite of this, this theory is not free from critics' eye sight. Critics have highlighted different drawbacks of this theory. The profounder of identity theory also boldly faced the problems and have given solution. But as the space of this paper is limited, so I will try to limit myself to analyse only two problems, viz., the problem of identity and the problem of co-existence. Finally effort will be made to give solution of these problems.

This paper is divided into two sections. In the first section of the paper I will narrate the problem of identity and the problem of co-existence. In the second section I will highlight the solution of the problems from the materialistic point of view.

\section{Section 1}

\subsection{The Problem of Identity}

There is a serious objection against the mind-brain identity theory which has not been satisfactory resolved. This problem concerns various non-intentional properties of mental states on the one hand and physical states on the other. For example, after images may be green or purple in colour, but nobody could reasonably claim that states of the brain are green or purple. Moreover, it may be the case that with a fair degree of accuracy brain states are spatially located where mental states are traditionally assumed as non-spatial.

Another problem of the theory is that it fails to give a satisfactory answer to the question of the relation and difference between mental and physical. 
Hillary Putnam challenges the identity theory from consideration of multiple realizibility. According to him, 'pain' is experienced not only by humans but by many different species of animal. But it seems unlikely that all these diverse organisms with the same pain experience are in the same identical brain state. And if the latter is the case, then pain cannot be identical to a specific brain state.

J. R. Searle puts forward certain problems for the identity theory. He states that identity theory violates the principle of logic called "Leibnitz Law". According to this law, any two things are called identical if and only if all the properties which they bear are found common to both of them. This means that the properties which we find in one thing are also the properties which we find in another thing. If this law is violated, that is, if it is possible to show that the properties of mental state cannot be attributed to brain and vice-versa then it refutes the identity theory. In this connection Searle cites an example-

"So I can say, for example, that the brain state that corresponds to my thought that it is raining is $3 \mathrm{~cm}$ inside my left ear; but, according to the objectors, it does not make any sense to say that my thought that it is raining is $3 \mathrm{~cm}$ inside my left ear. Furthermore, even for conscious states that have a location, such as pain, the pain may be in my toe, but the brain state that corresponds to that pain is not in my toe, but in my brain. So the properties of the brain state are not the same as the properties of the mental state. Therefore, Physicalism is false".

There is another objection against the identity theory. if the identity of mental states and brain states are empirical one, that is if the identity is discovered empirically, for example, if it could be discovered on the analogy with water and $\mathrm{H}_{2} \mathrm{O}$, or lightning and electrical discharge, then it seems that there would have to be two kinds of properties which will define the two sides of identity statement. And these two kinds of properties must identify one and the same thing. Thus when it is said by the identity theorists that 'lightning is identical with an electric discharge' they must have to identify one and the same thing in terms of the properties of lightning and the properties of electrical discharge, or when it is said that 'water is identical with $\mathrm{H}_{2} \mathrm{O}$ molecules' has to identify one and the same thing in terms of the properties of water and the properties of $\mathrm{H}_{2} \mathrm{O}$. Thus when the identity theorists claim that 'pain is identical with a certain types of brain state' they have to identify one and the same thing in terms of the properties of pain and the properties of brain-state. But problem arises if it is believed that in the identity statement there are two sets of properties and these properties are independent. In that case it appears that there are two different types of properties - mental properties and physical properties. And this belief of two sets of properties takes us back into property dualism.

Again, if it is true that all mental states are brain states, then what appears is that brain states are of two kinds - mental states and non-mental states. The mental states are of brain have mental properties and those of non mental states of brain have only physical properties. If it is so, then it sounds like property dualism.

Another objection slightly more technical was labeled against the identity theory and this was the accusation of 'neuronal chauvinism'. This objection really vibrated the identity theorists and they were indeed forced to do some modification in their theory. This charge was highlighted by Searle and says-

"If the claim of the identity theorists was that every pain is identical with a certain kind of neuronal stimulation, then it seems that a being that did not have neurons or that did not have the right kind of neurons could not have pains and beliefs. But why cannot animals that have brain structures different from ours have mental states? And indeed, why could not we build a machine that did not have neurons at all, but also had mental states?"

Searle claims that facing this objection the identity theorists are bound to shift from what is called 'type-type identity theory to 'token-token identity theory'. Thus in order to establish this claim Searle explains the distinction between type and token. Write the word "cow" three times: "cow" "cow" "cow". A question arises whether one word or three words are written. It is not debatable that here one type of word is written in three instances, or three tokens of one word is written. By types he means abstract general entities and by tokens he illustrates those which are concrete particular objects and events of those abstract generals. Thus when it is said that 'a token of a type' it means that it is a particular concrete example of abstract general type.

Let us now see how Searle proves that the identity theorists are moved from a type-type identity theory to a token-token identity theory. According to him, it is the point of the type-type identity theory that every type of mental state is identical to some type of physical state. Searle claims that by their own assertion it is a bit sloppy of the identity theorists. Because the identity in question is not between abstract universal types but between actual concrete tokens. What the identity theorists mean is that for every mental-state type there is some brain-state type and thereby every token of the mental type is nothing but a token of the brain type. The simple version of the token identity theorists, according to Searle, is-

"for every token of a certain type of mental state, there is some token of some type of physical state or other with which that mental state token is identical. They, in short, did not require, for example, that all token pains had to exemplify exactly the same type of brain states even though they were all tokens of the same mental type, pain. For that reason they were called 'token-token' identity theorists as opposed to 'type-type' identity theorists." 
Searle claims that token-token identity seems to be more plausible than type-type identity. In this connection he cites an example that two persons may have same belief that 'Denver is the capital of Colorado but it is not necessary to suppose that they have exactly the same type of neurobiological state. The neurobiological state of one's belief might be at a certain point of his brain and another's might be at another point although they have the same belief.

Searle thinks that in giving examples the identity theorists are often found very weak and this is unfortunate. Although they have given different examples, the favorite one is that pains are identical with Cfibre stimulations. But in giving this example both 'type-type' and 'token-token' identity theorists differ in certain extent. The former believes that every pain is identical with some $\mathrm{C}$-fibre stimulation where as the latter believes that not every pain but particular pain might be identical with particular C-fibre stimulation. Regarding other pains they think that this might be identical with some other state of a brain or machine. But all these are designated by Searle as 'bad neurophysiology'. In explaining the status of C-fibre Searle says that C-fibres are a type of axon which carries certain types of pain signals to the brain. Pain mechanism is a complex one in the brain and nervous system and C-fibre is just a part of this complex mechanism. Thus neurophysiologically it would be ridiculous to think that except C-fibre stimulation, there is nothing in the pain. Thus there is a good deal of debate centered round the question whether or not we would get such type of identity as exemplified by the type-type identity theorists. Or is it token identity upon which we could hope for. Searle believes that in between type and token identity, it is latter one which is more influential than the former.

In spite of its acceptability the token identity theorists have been facing another question and this is the question of commonness. What common things are there in all of these tokens which make the same mental state type? If it is believed by both $\mathrm{X}$ and $\mathrm{Y}$ that Denver is the capital of Colorado then apart from their brain state what exact thing is common in them but again both $\mathrm{X}$ and $\mathrm{Y}$ have different types of brain states? There are two answers that we find traditionally-one is from the dualist's quarter and another from type-type. But none of these answers will do for the token physicalist. Because the whole idea of the token identity theorists is to eliminate any type of irreducible mental properties and as such they cannot accept that the common thing in between $\mathrm{X}$ and $\mathrm{Y}$ is same irreducible mental properties. Again, the token theorists cannot accept the view that the same type of brain state is common in $\mathrm{X}$ and $\mathrm{Y}$ because it is this point disagreeing upon which there is move from type identity to token identity. Thus the answer that a particular mental state type and certain brain state type are identical cannot save the identity theorists from their downfall.

Soul Kripke also raised an objection against type-type identity theory from consideration of rigid reference. The identity theorists claim that the identity of mental and brain states are contingent. But Kripke argues that this identity is necessary, if true. He introduces two types of designators of entity, namely, rigid and non-rigid designators and holds that rigid designators refer to the same entity in every possible world and therefore identity of two entities referred by two rigid designators are necessary. Non-rigid or flaccid designators may refer to different entities in different possible worlds and identities of their referents thereby are contingent. The expression 'Benjamin Franklin' which always refers the same person is a rigid designator. The expression 'The inventor of daylight saving time' which, according to him, is a non rigid designator, although this expression refers to Benjamin Franklin in the actual world. In a possible world Benjamin Franklin may not be the inventor of daylight saving time. Anybody else other than Benjamin, the actual inventor, might have been the inventor of daylight saving time. On the other hand, it is not the case that someone else, other than Benjamin Franklin, might have been Benjamin Franklin. It is due to this reason Kripke says that 'Benjamin Franklin' is a rigid designator, where as 'the inventor of daylight saving time' is non rigid.

Thus with these two types of designators Kripke examines the mind-body identity statement. He claims that if one term is rigid and another is non rigid in an identity statement, the statement is not necessarily true and it might turn out to be false. Thus the statement 'Benjamin Franklin is identical with the inventor of daylight saving time' is true no doubt but this truth is contingent one because there may be a possible world where this statement is false. Kripke says that a statement must be necessarily true if it is the case that both sides of the identity statement are rigid and the statement is true. In this connection he cites the statement 'Samuel Clemens is identical with Mark Twain' and says that this statement is necessarily true because here both sides of the above statement mean one and the same person. It is impossible to imagine that there is a world where Samuel Clemens exists and also Mark Twain exists but they are not one and the same person but two different individuals. This is also true in the case of words that name natural kinds of things, for example, the statement 'water is identical with $\mathrm{H}_{2} \mathrm{O}$ '. Here both the expressions 'water' and ' $\mathrm{H}_{2} \mathrm{O}$ ' are rigid and the stament is true, therefore, this identity is necessary. Kripke finds the relevancy of this kind of argument in the case of mindbody problem. He says that if it is found that both the expressions 'mental state' and 'brain state' refer rigidly and the identity statement containing those expression is true then the statement must be necessarily true. Thus 'pain is equivalent to C-fibre stimulation' would have to be necessarily true if it is the case that pains were really identical with C-fibre stimulations. Here all these depend on the condition that if it were to be true at all. But Kripke claims that this statement is not necessarily true. Because it can be imagined that there exists pain 
without a C-fibre stimulation and also C-fibre stimulation without pain, although pains and C-fibre stimulations have strict correlations in this world. Thus Kripke logically concludes that if the statement 'pain is identical with C-fibre stimulation' is not necessarily true on the ground mentioned above then it cannot true at all, and hence it is false. His suggestion regarding identification of pains and neurobiological events is that there is a hope for identity theory if it is really the identification of particular conscious mental states and physical events.

\subsection{The Problem of Co-existence} existence.

Another problem that has been raised by the critics of the identity theory is the problem of co-

This problem is discussed by Shaffer (1994) from two standpoints: co-existence in time and coexistence in space. According to him, if two things are really to be identical then they must fulfil the conditions of co-existence in time and space. Two things which are apparently different may be one and the same; subject to condition that they must exist at the same time and in the same space. For example, whether Mr. X and Mr. Y are two different individuals can be assessed by ascertaining whether Mr. X existed in a particular location where Mr. Y was absent or whether Mr. X existed in a particular time when Mr. Y was absent. A person who was blamed for an offence can be declared innocent only by virtue of the fact that he was not there at the time of the offence and hence he cannot be the offender. It is undoubtedly true that the person who stole the diamond in Chicago and Mr. X are certainly not one and the same person if Mr. X was not in Chicago at the time when it was stole.

Mental events and physical events are said to be identical if and only if they meet the conditions of coexistence of both time and space. Now I will scrutinize the identity theory and see whether this theory fulfils the condition of co-existence.

Shaffer admits that we have very little knowledge about the co-existence in time. So far we have facts in our hands is only from the brain surgeon who is capable to stimulate the exterior part of the brain during surgery. He says that in many cases at the moment of brain operation, a patient may be fully conscious as because at that time only a local anaesthetic is necessary. Thus the brain patient may report the happenings of different mental events, such as, memories, thoughts, and sensations etc. during the stimulation of the different parts of the brain by the surgeon. Now question arises whether both physical events in the brain and mental events occur exactly at the same time or not. It is certainly impossible at present to answer it rightly. To prove that both physical events and mental events were not identical, it is only a very small gap in time is required. But the more difficult task is to establish that there is really a small gap in that case. Supposing that a small time-gap is established but yet it would not be correct to generalize that mental event and physical events are not identical. At best it can be said that the mental event was not identical with just that physical event. Thus Shaffer believes that as there is no possibility to have conclusive information from this sort of empirical work just described, so for long time there is no fear of refutation for the identity theorists from this quarter.

So far I have discussed above the problem of co-existence of time as analysed by Shaffer and it is evident from this analysis that identity between mental events and physical events cannot be fully accepted or rejected outright. Now effort will be made to see whether co-existence in space is possible given the identity theory. Shaffer is of the opinion that this task is also not so easy. It is very difficult to answer whether mental events and physical events occur in the same space or not. Shaffer believes that there are mainly two reasons for which the difficulty arises. These are:

1. Regarding knowledge of neurophysiology we are yet not fully aware. We are almost ignorant about the nature and function of the brain for which it is not possible to localize the relevant physical events. At best we can say that physical events are located in the brain and not much more than that known even up to present day. It may be that in the near future we will be able to learn much more about that.

2. Regarding the location of mental events we are also not fully clear for which it is difficult to say that there is identity in space of both mental events and physical events. It is very difficult to answer whether the mental events, such as, thought, feelings and wishes occur in the brain or any other places. If someone thinks something and if he is asked to answer the exact location where his thinking occurs then the obvious answer would be that it occurs in the place wherever the person concerned is when he has that thought. If he is in the college when he thinks, then his thought occurs in the college and nowhere else. But to ask the exact location of his interior part of the body where his thought occurs, whether it is in foot or liver or heart or head, is utterly unnatural. Because no one of these places is a location where thought occurs and therefore, preference of one place than another is a wrong answer. But all these do not mean that other than foot, liver, heart or head, our thought occurs somewhere else of our body. It is rather nonsense to say that somewhere else in our body the thought occurs. Someone may point a place of his body and claim that on that point his entertaining thought was located. But this claim is difficult to understand. Because it is certain that observing thoroughly the claimed point one would not be able to see anything resembling a thought. In reply to this point the identity theorists may further claim that pain can 
be located in the body but it cannot be seen. But this claim paves the answer that pain can be felt by someone but it is rare case that some one feels a thought in the body.

From the above analysis Shaffer says that as it is meaningless to say that in some point of body mental events occur, so it proves that identity theory cannot be true. If it is claimed that physical events occur somewhere within the body and if it is really true that these physical events are identical with mental events then logically it might be concluded that these mental events must occur in some point within the body. But, according to Shaffer, it is senseless to say that mental events occur here or there within the body because these actually do not occur at any point within the body. Thus he says that as mental events fail to fulfil the condition of co-existence in space and therefore in no way these can be identical with physical events. Shaffer says that we are unable to find out the location of both mental events and physical events in our body. But these inabilities in two cases are different. In the case of physical events, particularly of brain events, our knowledge is not so enough. But it may be assumed that after some days we will be able to know the exact location of relevant physical events. But this cannot be said in the case of mental events. There is no hope that in future our knowledge about the location of mental events will increase. To discover the location of thought in our brain is really a difficult task. There is no means by which we can find out the exact location of thought in our brain. It may be that every event that occur in our brain is understood by means of $\mathrm{x}$ - ray or by other means, but these means in no way can give us a glimpse about thought. To solve the problem it may be imagined that brain could be enlarged or shrink where we can roam about freely but in that way also we will not be able to find out the location of thought. It is only physical events that we will observe occurring in the brain. Thus Shaffer says,

"If mental events had location in the brain, there should be some means of detecting them there. But of course there is none. The very idea of it is senseless".

\section{Section 2}

\subsection{Resolution of the Problem of Identity}

So far I have delineated the problems of identity and the problem of co-existence that have been raised by different thinkers against the identity theory of mind. Now I will try to resolve these problems from the materialistic point of view.

'Leibniz's Law.'

Searle points out that Identity theory violates the principle of logic. By this principle he means

But in response to this objection they hold that this objection is based on the ignorance about the details of the brain. When a person comes to know more and more about the brain, he will be able to attribute perfectly and comfortably the spatial location of mental states and at the same time to attribute the so called mental properties to the states of the brain. The identity theorist further says that in explaining the location of the pain in the toe, the totality of the neuro-physiological aspect of having the pain should be taken into account. This experience comprises the stimulation of the peripheral nerve endings in the toe up to the brain itself. Searle thinks that the answer given by the identity theorist to the objection is a satisfactory one but there are more serious objections which they have to face.

Searle raises another objection that the identity theory sounds like property dualism. But for the identity theorists the problem is a decisive one. The whole point of their theory is to save materialism. The central point of their theory is to show that mental states are really identical with the states of the brain, that is, mental states are reducible to material states of the brain. But if this theory admits that mental states are irreducible to brain states then the whole project of their theory fails and it will leave an irreducible mental element for us. In order to avoid such consequence the identity theorists propose that without using any mental predicates the phenomena in question can be specified. They can be specified in a topic-neutral vocabulary. In explaining this specified topic-neutral vocabulary they say that instead of saying that 'there is a yellow-orange afterimage in me' it is better to say that "there is something going on in me that is like what goes on when I see an orange". Thus the second objection was supposed to be answered by rephrasing the identification of the mental state in a 'topic-neutral vocabulary. This topic-neutral vocabulary enables us to specify the mental element in a non-mental, neutral vocabulary, e.g., 'there is this thing going on in me'. This statement can be more specified in a neutral way without being committed to dualism or materialism. Here the thing in question is a brain process. Thus in consistent with materialism, mental features can be specified by us.

Searle is not satisfied with this answer. He says that the answer given by the identity theorists fail to convince the critics. To him, it is a fact that mental phenomena continue to have mental properties. This fact does not change when we talk about mental phenomena without using a mental vocabulary. In this connection he cites the example of yellow-orange after image and says that this image remains a qualitative and subjective one and does not depend on our choice to mention those features. One may refuse to talk about airplanes and can just say that there are some properties which belong to United Airplanes. But this refuse of airplanes in no way eliminates the existence of airplanes.

We shouldbe focused on what exactly the identity theorist is proposing. He is not denying the 
existence of mental phenomena. What he suggests is to reject the dualistic framework, whether the Cartesian type or property dualism type. To insist upon irreducible mental entities or properties is a sign for a kind of spiritual quest that would never end. A more promising line of enquiry is the materliastic one and the dominant epistemological perspective should come from third person perspective supplemented by the first person perspective. The stance should not be that either this perspective or that.

The third objection comes from the accusation of 'neuronal chauvinism'. This objection comes from a readiness to accept a stubborn dualism which the identity theorist is painstakingly advocating to drop.

The strength of Kripke's arguments rests on the assumption that the identity statement about mental state and brain state does not contain two rigid designators. 'Gold' is a rigid designator; 'the yellow metal' is not a rigid designator and 'the element with atomic number 79' is a rigid designator. Hence, it is possible that there is gold without being the yellow metals where as it is impossible for gold not to be the element with the atomic number 79 . Gold being the element with atomic number 79 is a scientific discovery. Now suppose that the concepts 'mental state' and 'brain state' are well defined. Further assume that we have a scientific breakthrough and the identity of mental state and brain state is discovered. In that case it would be impossible for a mental state not to be a brain state. Expressions expressing those would be now regarded as two rigid designators.

\subsection{Resolution of the Problem of Co- existence.}

Regarding the problem of co-existence J. A. Shaffer mentions that the co-existence is of two types: coexistence in time and co-existence in space and he believes that the identity theory fails to convince us how the conditions of co-existence in time and space can be met.

But in reply to the problem of co-existence it is believed by some supporters of the identity theory that this objection can be met. They put forward two approaches in this connection. The first approach of them is that this objection of co-existence begs the question. It is paradoxical to ask that if mental events and physical events are really identical then mental events must occur in a location as the physical events occur and the location of mental events must be exactly in the same location where physical events occur.

Secondly, to reply the objection of co-existence, the identity theorists approach that it is the whole body where all the physical events occur and the occurrence takes place where the whole body is located. And the mental events also occur in the location where the body is located and in no way it is located in any particular part of the body. I think that the answer to the objection raised by Shaffer against the identity theory is satisfactory.

Shaffer declares the impossibility of identity theory on the alleged ground that by analytic necessity experiences are unlocated whereas neural events are located. These are located in part of the subject's neural system. But David Lewis severely criticized this view and says that there is no strength in this argument. According to Lewis, it is metaphysical prejudice to believe the principle that as experiences are unlocated so these enjoy analytic or other necessity and as such this claim cannot be respected. He says that if there is a way in which experiences are, by analytic necessity, unlocated, then this way is also irrelevant. Because in a primary sense we regard only concreta as allocated somewhere and this is perhaps in our pre-systematic thought. Abstracta are said to be located only in a derivative sense. These are located in concreta by the relation of inherence. Thus Lewis says,

"But this possible source of analytic unlocatedness for experiences does not meet the needs of Shaffer's argument. For neural events are abstracta too. Whatever unlocatedness occurs to experiences not because they are mental but because they are abstract must occur as much as to neural events. So it does not discriminate between the two."

\section{Conclusion}

In modern period the mind -body problem was introduced as a problem by Rene Descartes. He tried to solve the problem metaphysically and that is why failed to succeed in his mission. The theory of Behaviourism also attempted to solve the problem by denying the mental episodes. Thus dualism is right when it holds that mental items are inner but it is wrong when states that mental items are non-physical in nature. Similarly, behaviourism is right in its materialism but wrong when this theory repudiates inner mental episodes. Thus here identity theory is delightful synthesis of both dualism and behaviourism.

From the above analysis of the problems of the Identity Theory and their resolution I can conclude that in spite of its limitations in describing the mind-brain relation, this theory is better than all other theories on this issue. The profounder of this theory very logically answered the problems raised by the critics of the theory. This theory attempts to solve the problem scientifically and that is why this theory is more acceptable. 


\section{References}

[1]. D.MArmstrong, Bodily Sensations, (London, Routledge1961).

[2]. D.MArmstrong, A Materialist Theory of the Mind, (London, Routledge. 1993Second Edition).

[3]. D.M.Armstrong, The Headless Woman Illusion and the Defence of Materialism, Analysis, 29, 1968b, 4849.

[4]. S.Kripke, Naming and Necessity, (Cambridge, Mass., Harvard University Press, 1980).

[5]. D.Lewis, How to Define Theoretical Terms, Journal of Philosophy, 67, 1970, 427-446.

[6]. D.Lewis, Psychophysical and Theoretical Identifications, Australasian Journal of Philosophy, 50, 1972, 249-258.

[7]. D.Lewis, Philosophical Papers, Vol. 1, Oxford, Oxford University Press, 1983.

[8]. U.T. Place, Identifying the Mind, (New York, Oxford University Press, 2004).

[9]. J.Searle, ,Mind, A Brief Introduction, (New York, Oxford University Press, 2004)

[10]. J.J.C. Smart, On Some Criticisms of a Physicalist Theory of Colour, in Chung-ying Cheng (Ed.),Philosophical Aspects of the Mind-Body Problem, (Honolulu, University of Hawaii Press, 1975)

[11]. J.J.C.Smart, The Content of Physicalism ,Philosophical Quarterly, 28, 1978, 339-341. 